\title{
Sirt1 coordinates with ERa to regulate autophagy and adiposity
}

\author{
Zhipeng Tao ${ }^{1,6}$, Limin Shi', Jane Parke², Louise Zheng ${ }^{1}$, Wei Gư ${ }^{3}$ X. Charlie Dong ${ }^{4}$, Dongmin Liu', Zongwei Wang ${ }^{5}$,
} Aria F. Olumi ${ }^{5}$ and Zhiyong Cheng ${ }^{1,2}$

\begin{abstract}
Sex difference in adiposity has long been recognized but the mechanism remains incompletely understood. Previous studies suggested that adiposity was regulated by autophagy in response to energy status change. Here, we show that the energy sensor Sirt1 mediates sex difference in adiposity by regulating autophagy and adipogenesis in partnership with estrogen receptor a (ERa). Autophagy and adipogenesis were suppressed by Sirt1 activation or overexpression, which was associated with reduced sex difference in adiposity. Mechanistically, Sirt1 deacetylated and activated AKT and STAT3, resulting in suppression of autophagy and adipogenesis via mTOR-ULK1 and p55 cascades. ERa induced Sirt1 expression and inhibited autophagy in adipocytes, while silencing Sirt1 reversed the effects of ERa on autophagy and promoted adipogenesis. Moreover, Sirt1 deacetylated ERa, which constituted a positive feedback loop in the regulation of autophagy and adiposity. Our results revealed a new mechanism of Sirt1 regulating autophagy in adipocytes and shed light on sex difference in adiposity.
\end{abstract}

\section{Introduction}

Autophagy plays a central role in cellular repair, remodeling, development, and homeostasis ${ }^{1-3}$. Autophagy is upregulated during adipocyte differentiation, and inhibition of autophagy suppresses adipogenesis ${ }^{4-8}$. In obese or diabetic individuals, adipose autophagy was shown to be aberrantly activated ${ }^{9,10}$, while targeted suppression of autophagy in the adipose tissue protected against obesity ${ }^{4,5}$. These findings underscore the role of autophagy in adiposity regulation, but the mechanism has not been fully understood and the complexity can be increased by stress conditions (e.g., malnutrition, inflammation, and oxidative stress) ${ }^{11-13}$.

Sirtuin 1 (Sirt1) is an energy sensor that regulates metabolism across tissues ${ }^{14,15}$. Activation or overexpression of Sirt1 improves systemic metabolism and

\footnotetext{
Correspondence: Zhiyong Cheng (z.cheng@ufl.edu)

${ }^{1}$ Department of Human Nutrition, Foods, and Exercise, Virginia Tech,

Blacksburg, VA 24061, USA

${ }^{2}$ Food Science and Human Nutrition Department, University of Florida,

Gainesville, FL 32611, USA

Full list of author information is available at the end of the article

Edited by Inna Lavrik
}

protects against diabetes, obesity, or high-fat diet-induced metabolic damages ${ }^{16-22}$, while dysregulated Sirt1 resulted in phenotypes associated with diabetes, obesity, and aging $^{23}$. In adipocytes, upregulation of Sirt1 enhances lipolysis and attenuates adipogenesis ${ }^{24}$, and ablation of Sirt1 promotes adipocyte differentiation and increases adiposity in mice ${ }^{25,26}$. The roles of Sirt1 in white adipose tissue (WAT) development, maintenance, and remodeling, have been linked to negatively modulating adipogenesis via peroxisome proliferator-activated receptor gamma (PPAR $\gamma)^{24}$, enhancing oxidative phosphorylation via peroxisome proliferator-activated receptor gamma coactivator 1 -alpha $(\mathrm{PGC}-1 \alpha)^{16-19}$, potentiating brown adipose tissue function ${ }^{22}$ or inducing the browning of subcutaneous WAT in response to cold exposure ${ }^{27}$.

Sirt1 was shown to promote or suppress autophagy, partly because of Sirt1 deacetylating autophagy proteins such as Atg5, Atg7, or LC3 ${ }^{28-30}$. However, it is unknown whether and how Sirt1 interacts with autophagy in the regulation of adipogenesis and adiposity. In the present study, we investigated the effects of gain and loss of Sirt1 on autophagy and adiposity. We found that the mice with

\section{(c) The Author(s) 2021}

(c) (i) Open Access This article is licensed under a Creative Commons Attribution 4.0 International License, which permits use, sharing, adaptation, distribution and reproduction cc) in any medium or format, as long as you give appropriate credit to the original author(s) and the source, provide a link to the Creative Commons license, and indicate if changes were made. The images or other third party material in this article are included in the article's Creative Commons license, unless indicated otherwise in a credit line to the material. If material is not included in the article's Creative Commons license and your intended use is not permitted by statutory regulation or exceeds the permitted use, you will need to obtain permission directly from the copyright holder. To view a copy of this license, visit http://creativecommons.org/licenses/by/4.0/. 
increased expression of Sirt1 showed reduced adiposity, to a greater extent in females than males. Sirt1-induced reduction of adiposity was associated with lower autophagy activity, and the sex difference in adiposity change may be ascribed to the crosstalk between estrogen receptor ER $\alpha$ signaling and Sirt1-autophagy axis. Mechanistically, Sirt1 deacetylated AKT and STAT3, which activated mTOR-ULK1 and STAT3-p55 (p55 subunit of phosphoinositide 3-kinase) signaling pathways, respectively, to mitigate autophagy in adipocytes. ER $\alpha$ and Sirt1 form a positive feedback loop that enhances the effects on autophagy and adiposity. Our study unraveled a new mechanism of Sirt1 regulating adiposity and sex difference through the crosstalk with $E R \alpha$ and autophagy.

\section{Results}

Sirt1 expression is negatively correlated with autophagy and adipogenesis

During adipogenesis, lipid accumulation was significantly increased (Fig. 1a). Compared with preadipocyte (day 0 ), Sirt1 expression was drastically reduced in mature adipocyte (day 12), paralleled with significant decrease in LC3-II (Fig. 1b), the substrate that is selectively degraded by lysosomal hydrolase in autolysosome ${ }^{31,32}$. Measurement of autophagy flux (i.e., contrasting the rates of removing substrates LC3-II and p62 by autophagy in the absence and presence of autophagy inhibitors bafilomycin A1 and leupeptin ${ }^{31}$ ) indicated higher autophagy activity in mature adipocytes than in preadipocytes (Fig. 1c). The inverse correlation between Sirt1 expression and autophagy activity suggests that Sirt1 suppresses autophagy, the cellular remodeling process required for adipocyte differentiation $^{4-8,33}$.

\section{The effects of gain of Sirt1 on autophagy and adipogenesis}

To examine whether the gain of Sirt1 suppresses autophagy in adipocytes, we overexpressed Sirt1 in 3T3L1 cells using knock-in technique. As shown in Fig. 2a, b, Sirt1 knock-in (Sirt1-KI) significantly increased the expression of Sirt1 protein in 3T3L1 cells, which largely suppressed adipogenesis even in the presence of differentiation inducer (DI). The mitigated adipogenesis was associated with reduced autophagy flux activity (Fig. $2 \mathrm{~b}, \mathrm{c})$. These findings support the notion that autophagy is required for adipogenesis (Fig. 1) (-8,33 $^{4-}$ and Sirt1 appears to be an autophagy suppressor in adipocytes. In addition, our data, along with the evidence from MEFs, stem cells, and cancer cells ${ }^{28-30}$, suggests that Sirt1 may play tissueor cell type-dependent roles in autophagy.

\section{The effects of loss of Sirt1 on autophagy and adipogenesis}

To test whether the loss of Sirt1 may increase autophagy activity and adipogenesis, we used RNA interfering

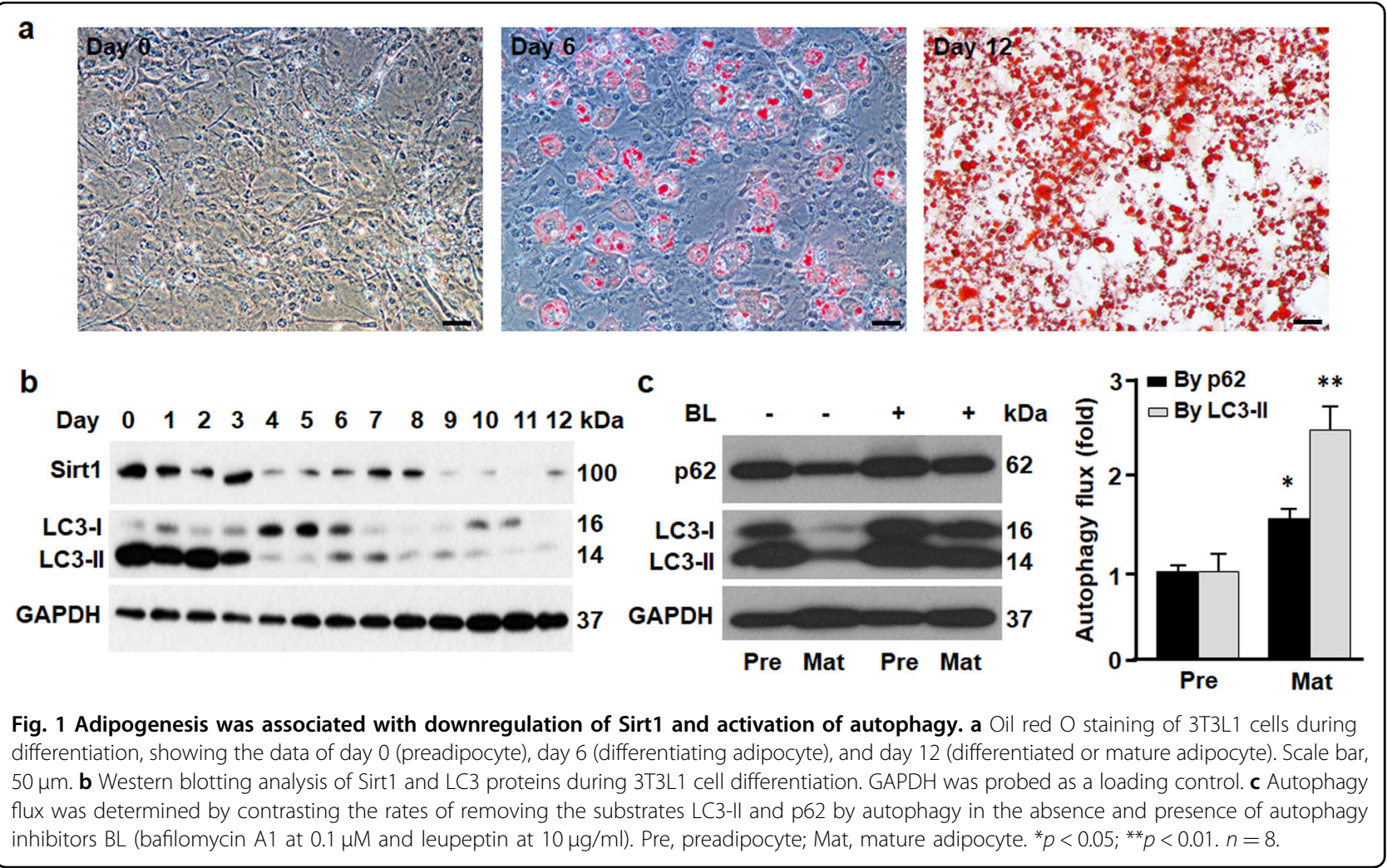



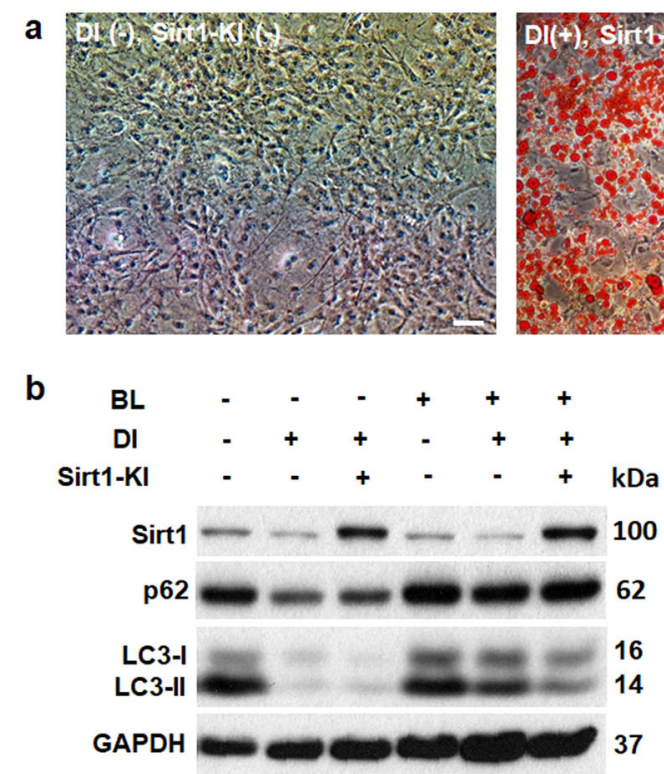
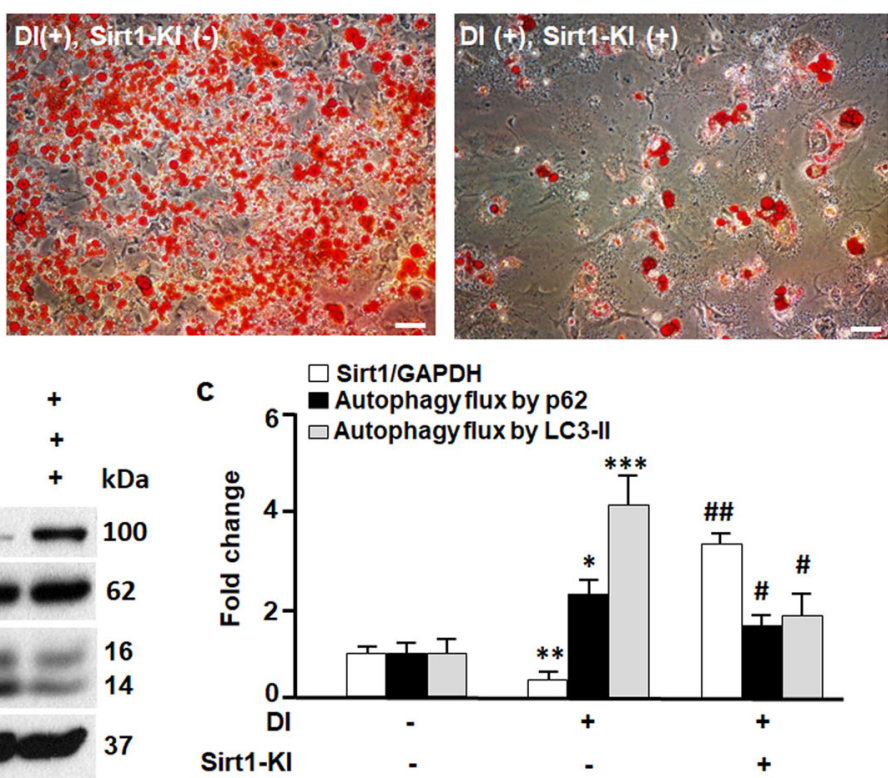

Fig. 2 The effects of gain of Sirt1 on autophagy and adipogenesis. a Oil red O staining of 3T3L1 cells on day 12, in the absence (-) or presence $(+)$ of differentiation inducer (DI) and Sirt1 knock-in or overexpression (Sirt1-KI). Scale bar, $50 \mu \mathrm{m}$. b Western blotting analysis of Sirt1, p62, and LC3 proteins. GAPDH was probed as a loading control. Autophagy inhibitors bafilomycin A1 at $0.1 \mu \mathrm{M}$ and leupeptin at $10 \mu \mathrm{g} / \mathrm{ml}$ (BL) were used to treat the cells and measure the turnover of autophagic substrates p62 and LC3-II (i.e., autophagy flux activities). c Densitometric analyses of Sirt1 and autophagy flux. Comparing DI(-)Sirt1-KI(-) with DI(+)Sirt1-KI(-): ${ }^{*} p<0.05 ;{ }^{* *} p<0.01 ;{ }^{* * *} p<0.001$. Comparing DI(+)Sirt1-KI(-) with DI(+)Sirt1-KI(+): ${ }^{\#} p<0.05 ;{ }^{\# \#} p<0.01 . n=8$.

technique to knock down Sirt1 (Sirt1-KD) in 3T3L1 cells (Fig. 3). As shown in Fig. 3a, Sirt1-KD promoted adipocyte differentiation, leading to a greater accumulation of lipid in 3T3L1 cells compared to the 3T3L1 cells incubated with differentiation inducer (DI) alone. Moreover, Sirt1-KD significantly increased autophagy flux activities (Fig. 3b, c). These results underscore Sirt1 as a key suppressor of autophagy during adipogenesis (Figs. 1-3).

\section{Sirt1 suppresses autophagy via mTOR-ULK1 pathway}

Autophagy is a multi-step process that includes initiation, membrane nucleation, expansion, fusion, and degradation ${ }^{34}$. Western blotting analysis suggested that modulation of Sirt1 had marginal effects on beclin 1, Atg5, Atg7, or Atg12-Atg5 conjugate (Fig. 1s), the proteins known to regulate membrane nucleation and expansion $^{34}$. However, overexpression of Sirt1 (Sirt1-KI) in 3T3L1 cells induced inhibitory phosphorylation of ULK1 (p-ULK1-Ser757), the protein that plays a key role in autophagy initiation (Fig. 4a, b). This may account for the suppressed autophagy in Sirt1-KI (Fig. 2). By contrast, knockdown of Sirt1 (Sirt1-KD) drastically attenuated pULK1-Ser757 (Fig. 4c, d), which was associated with deactivation of the serine/threonine protein kinase mTOR (i.e., dephosphorylation of mTOR at Ser2448) $)^{35,36}$. Consistently, Sirt1-KI significantly upregulated p-mTORSer2448, known to enhance mTOR kinase activity and suppress autophagy by phosphorylating ULK1 at Ser757 (Fig. 4a, b) ${ }^{37}$. To confirm the effects of Sirt1 on mTOR activity in vivo, we studied the adipose tissues from Sirt1 transgenic (S1tg) mice (Fig. 4e-g) ${ }^{20}$. Overexpression of Sirt1 activates mTOR by significantly increasing the phosphorylation level at Ser2448 (p-mTOR-Ser2448), which was associated with an elevation in mTORmediated inhibitory phosphorylation on ULK1 (p-ULK1Ser757). As the downstream targets of mTOR, the proteins p70S6K and 4EBP1 exhibited significantly higher phosphorylation levels in S1tg mice compared with the control mice (Fig. 4e-g) ${ }^{36,38}$. Therefore, Sirt1 activates the mTOR-ULK1 cascade to dampen autophagy.

\section{Sirt1 deacetylates and activates AKT and STAT3}

Previous studies showed that Sirt1 might regulate autophagy due to deacetylation of Atg5, Atg7, or LC3 in cancer cells, MEFs, germ cells, and stem cells ${ }^{28-30,39}$. Using immunoprecipitation and immunoblotting analyses, we pulled down acetylated proteins and probe Atg5, Atg7, and LC3 in adipose tissues from control and S1tg mice. Unexpectedly, S1tg and the control mice showed comparable acetylation in Atg5, Atg7, and LC3 (Fig. 2s). However, overexpression of Sirt1 drastically deacetylated protein kinase B (Akt) and signal transducer and activator of transcription 3 (STAT3) in S1tg mice (Fig. 4h-j). Deacetylation of Akt was shown to enhance its 
a
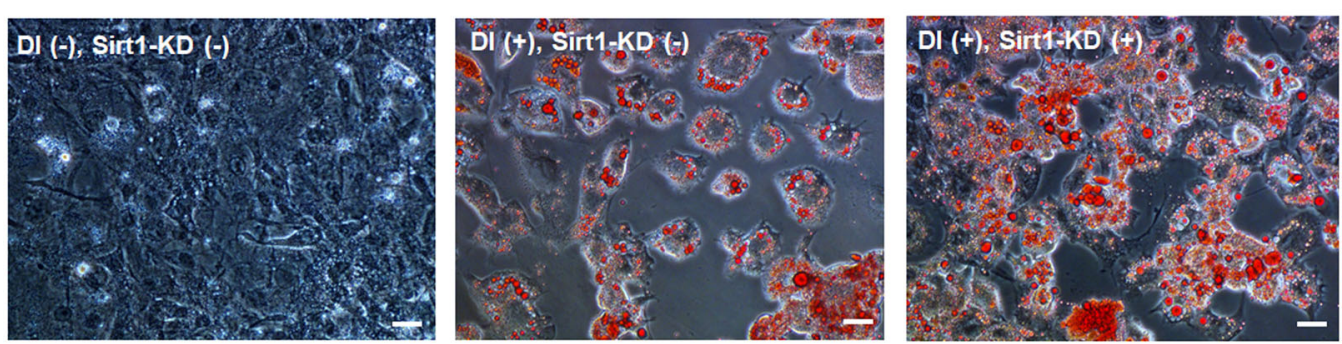

b

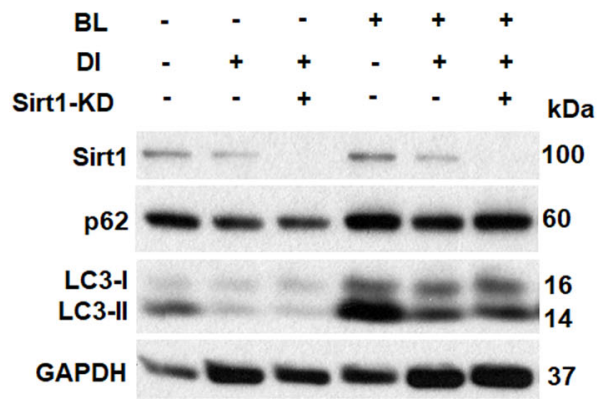

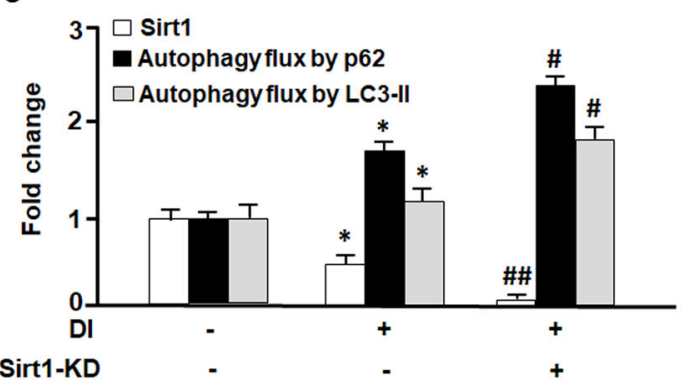

Fig. 3 The effects of loss of Sirt 1 on autophagy and adipogenesis. a Oil red O staining of $3 T 3 \mathrm{~L} 1$ cells on day 9 , in the absence $(-)$ or presence $(+)$ of differentiation inducer (DI) and Sirt1 knock-down (Sirt1-KD). Scale bar, $100 \mu \mathrm{m}$. b Western blotting analysis of Sirt1, p62, and LC3 proteins. GAPDH was probed as a loading control. Autophagy inhibitors bafilomycin A1 at $0.1 \mu \mathrm{M}$ and leupeptin at $10 \mu \mathrm{g} / \mathrm{ml}(\mathrm{BL}) \mathrm{were}$ used to treat the cells and measure the turnover of autophagic substrates p62 and LC3-II (i.e., autophagy flux activities). c Densitometric analyses of Sirt1 and autophagy flux. Comparing $\mathrm{DI}(-)$ Sirt1-KD(-) with $\mathrm{DI}(+)$ Sirt1-KD(-): ${ }^{*} p<0.05$. Comparing DI(+)Sirt1-KD(-) with $\mathrm{DI}(+)$ Sirt1-KD(+): ${ }^{\#} p<0.05$; ${ }^{\# \#} p<0.01 . n=8$.

phosphorylation and kinase activity in phosphorylating $\mathrm{mTOR}^{40}$, and deacetylation of STAT3 increases its transcription factor activity ${ }^{41-43}$. Indeed, Akt phosphorylation and p55 expression were markedly upregulated in S1tg mice in comparison to the control mice (Fig. $4 \mathrm{~h}-\mathrm{j}$ ). Consistently, phosphorylation of mTOR and its downstream target proteins $\mathrm{p} 70 \mathrm{~S} 6 \mathrm{~K}$ and $4 \mathrm{EBP} 1$ were induced in S1tg mice (Fig. 4e-g), supporting the notion that Akt activates $\mathrm{mTOR}^{44-47}$. Moreover, the activation of STAT3p55 cascade by Sirt1 may serve as an additional mechanism of the mitigated autophagy because p55 was depicted as an inhibitor of autophagy (Fig. $4 \mathrm{~h}, \mathrm{j})^{42}$.

\section{Sirt1 reduces sex difference in adiposity}

To examine how the Sirt1-autophagy axis affects adiposity in vivo, we measured the fat mass of S1tg mice and control mice (Fig. 5a, b). S1tg mice exhibited significantly lower fat mass compared with the control mice, in line with Sirt1 suppressing autophagy and adipogenesis in vitro (Figs. 1-3) and Sirt1 activation preventing adipose expansion in mice ${ }^{16,17}$. Intriguingly, the sex difference in S1tg mice was attenuated drastically in comparison to control mice (Fig. 5c). Western blotting analysis revealed that in control mice, females had a higher expression of Sirt1 than in males, while the difference was largely abolished by Sirt1 overexpression in S1tg mice (Fig. 5d). These findings suggested the Sirt1 might crosstalk with sex hormone signaling, particularly estrogen receptor ER $\alpha$ signaling, because ER $\alpha$ has been shown to regulate autophagy and adiposity ${ }^{7,48,49}$. Indeed, treatment of 3T3L1 cells with estradiol (E2) markedly induced Sirt1 expression (Fig. 5e), while knockout of ER $\alpha$ attenuated Sirt1 expression (Fig. 5f), underscoring Sirt1 as a downstream target of ER $\alpha$. When adipocytes were treated with E2, it increased phosphorylation of mTOR and ULK1 (Fig. $5 \mathrm{~g}, \mathrm{~h}$ ), the signaling pathway known to mitigate the initiation of autophagy (Figs. 1-4) $)^{7,34}$. In line with autophagy required for adipogenesis, E2 treatment substantially inhibited adipocyte differentiation (Fig. 5i). However, knockdown of Sirt1 (Sirt1-KD) abolished E2induced effects on the mTOR-ULK1 cascade and adipogenesis, further confirming that E2/ER $\alpha$ signaling regulates autophagy and adipogenesis by inducing Sirt1 expression (Fig. 5g-i). Therefore, the reduced sex difference in adiposity in S1tg mice may be explained at least in part by the fact that Sirt1 overexpression overrides the effects of E2/ER $\alpha$.

\section{Sirt1 deacetylates ERa and reduces adiposity}

Given the nature of Sirt1 as a deacetylase, we asked the question whether adipose ER $\alpha$ is deacetylated by Sirt1. Intriguingly, overexpression of Sirt1 significantly reduced acetylation level of ER $\alpha$ in S1tg mice in comparison to the control mice (Fig. 6a). Total ER $\alpha$ protein level was higher in S1tg mice than in the control mice, but the difference was not statistically significant (Fig. 6a). In mature 3T3L1 


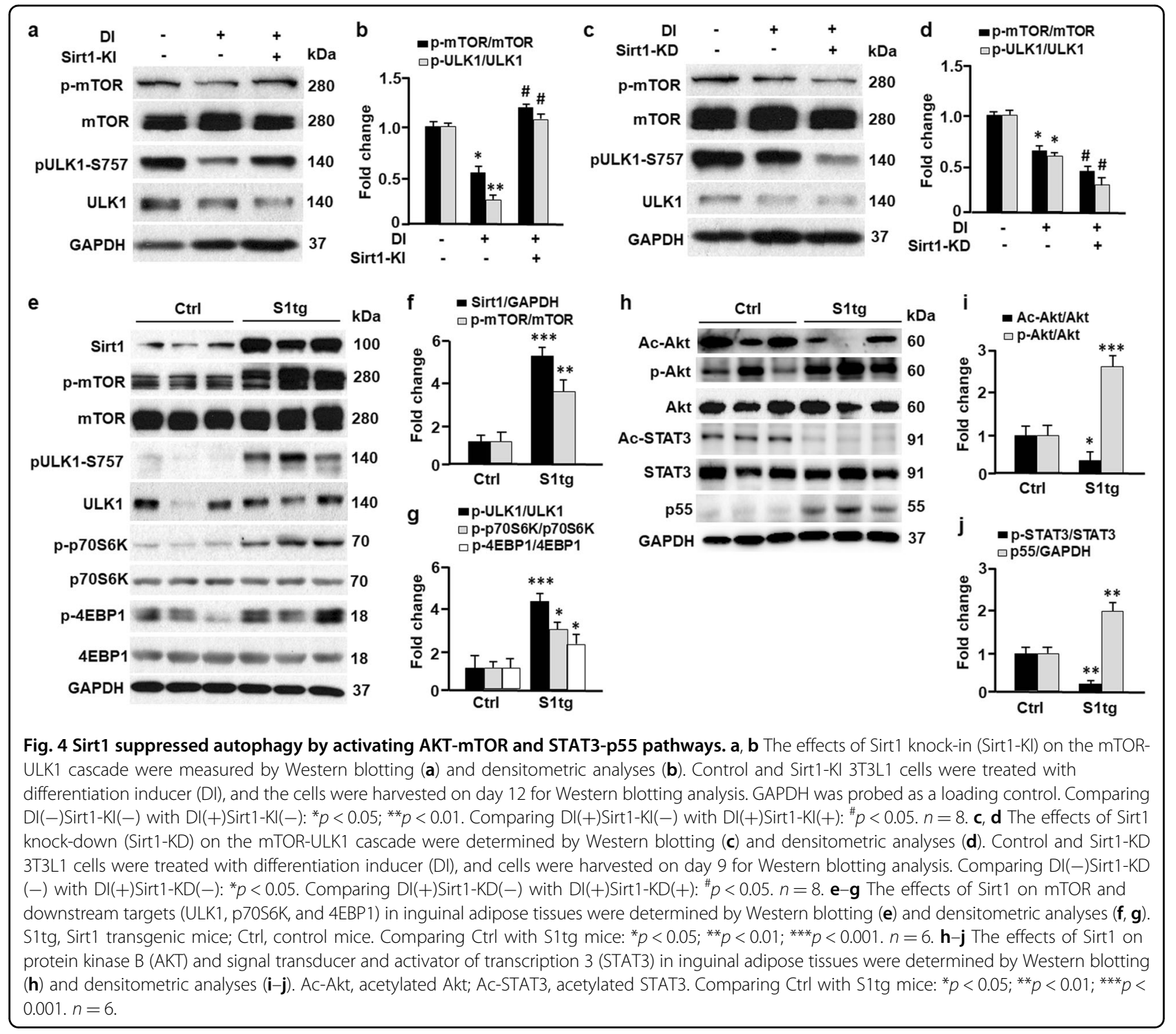

adipocytes (DI+/NMN-), acetylation of ER $\alpha$ increased by over twofold $(p<0.01)$ in comparison to preadipocytes $(\mathrm{DI}-/ \mathrm{NMN}-)$, as shown in Fig. 6b. Total ER $\alpha$ protein level was reduced in mature 3T3L1 adipocytes, consistent with the notion that suppression of ER $\alpha$ signaling promotes adipogenesis ${ }^{7}$. However, when 3T3L1 cells were treated with nicotinamide mononucleotide (NMN, $100 \mu \mathrm{M})$, a Sirt1 activator that promotes biosynthesis of $\mathrm{NAD}^{+15,50,51}$, it significantly reduced acetylation level of $\mathrm{ER} \alpha$ (Fig. 6b). These in vitro and in vivo data confirm that $\mathrm{ER} \alpha$ is deacetylated by Sirt1, suggesting that Sirt1 forms a positive feedback loop and promotes E2/ER $\alpha$ signaling cascade in the regulation of adiposity. In line with this, Sirt1 overexpression in males reduced adiposity by $0.7 \%$ (Fig. 6c); however, Sirt1 overexpression in females (known to have stronger $\mathrm{E} 2 / \mathrm{ER} \alpha$ signaling ${ }^{52,53}$ ) resulted in greater reduction of adiposity, i.e., by $2.3 \%$ ( $p<0.001$ vs. $0.7 \%$ in the males). Thus, our data support the model where E2/ ER $\alpha$ signaling induces Sirt1-mediated suppression of autophagy and adipogenesis, and Sirt1 forms a positive feedback loop to enhance E2/ER $\alpha$ signaling in the regulation of adiposity (Fig. 6d).

\section{Discussion}

The mechanism of sex difference in adiposity is complex and remains incompletely understood ${ }^{54,55}$. Previous research has implicated both sex chromosome and hormones in adipose development and growth ${ }^{7,54,55}$. In this study, we identified an E2/ER $\alpha$-Sirt1 axis that regulates autophagy and sex difference in adiposity. Activation of $E 2 / E R \alpha$ signaling induced Sirt1 expression (Fig. 5e, f), presumably through transactivation by binding to the 


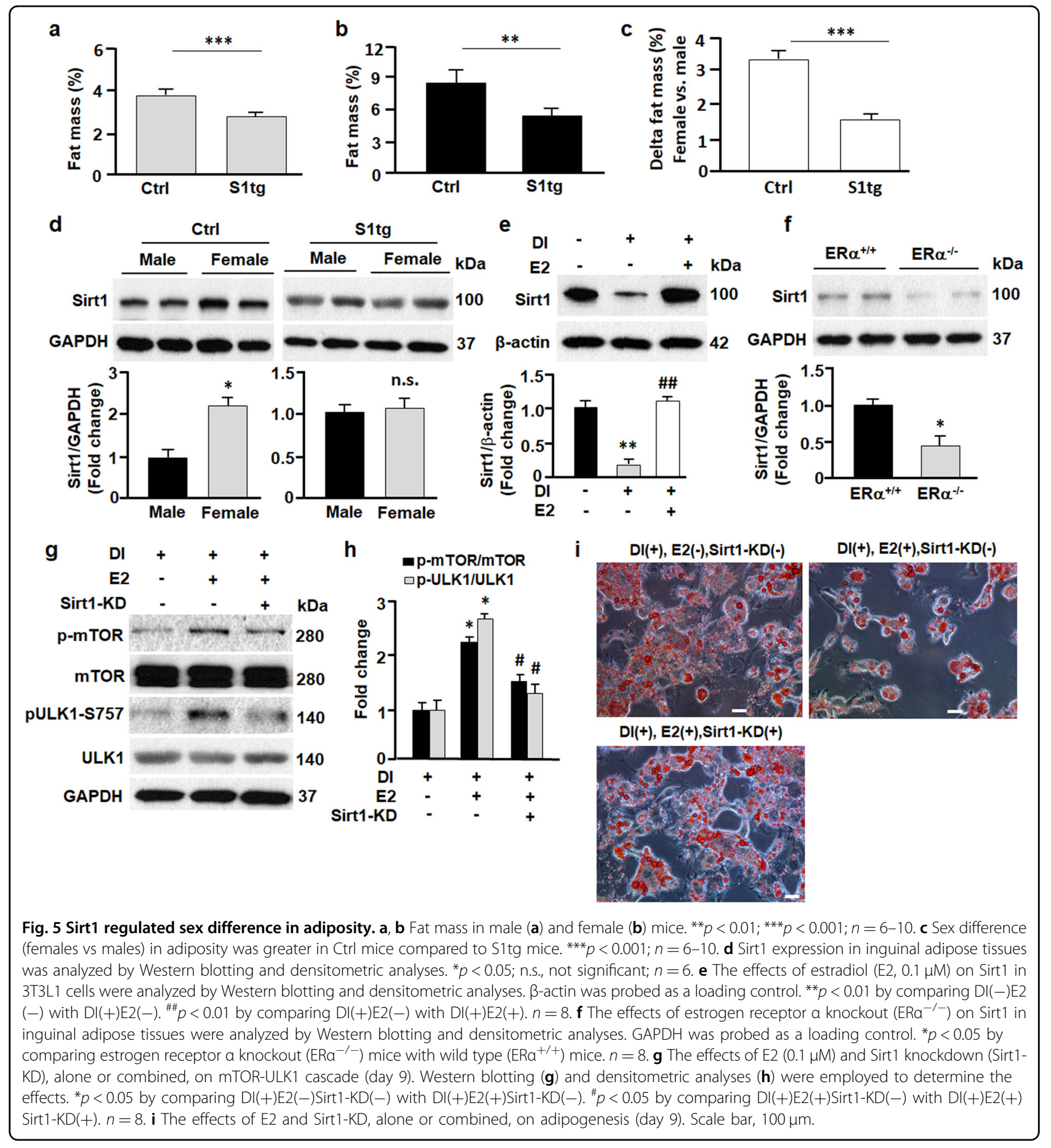

promoter of Sirt1 gene and increasing its transcription ${ }^{56}$. Overexpression of Sirt1 phenocopied the effects of E2/ $\mathrm{ER} \alpha$ signaling, during which mTOR-ULK1 cascade was activated and dampened autophagy and adipogenesis (Figs. 2, 4 and 5) ${ }^{7}$. Consistently, silencing Sirt1 abolished the effects of E2/ER $\alpha$ signaling on autophagy and adipogenesis (Figs. 3 and 5). In line with E2/ER $\alpha$ signal differing in males and females ${ }^{52,53}$, Sirt1 expression was higher in females vs. males in the control mice (Fig. 5d). However, the sex difference in Sirt1 expression was normalized in S1tg mice, and the sex differences in adiposity was significantly reduced in comparison to control mice $(p<0.001$, Fig. 5c).

Additional mechanism of Sirt1-induced suppression of autophagy includes the STAT3-p55 pathway. STAT3 was shown to disrupt lysosomal function ${ }^{57}$.The p55 subunit of 


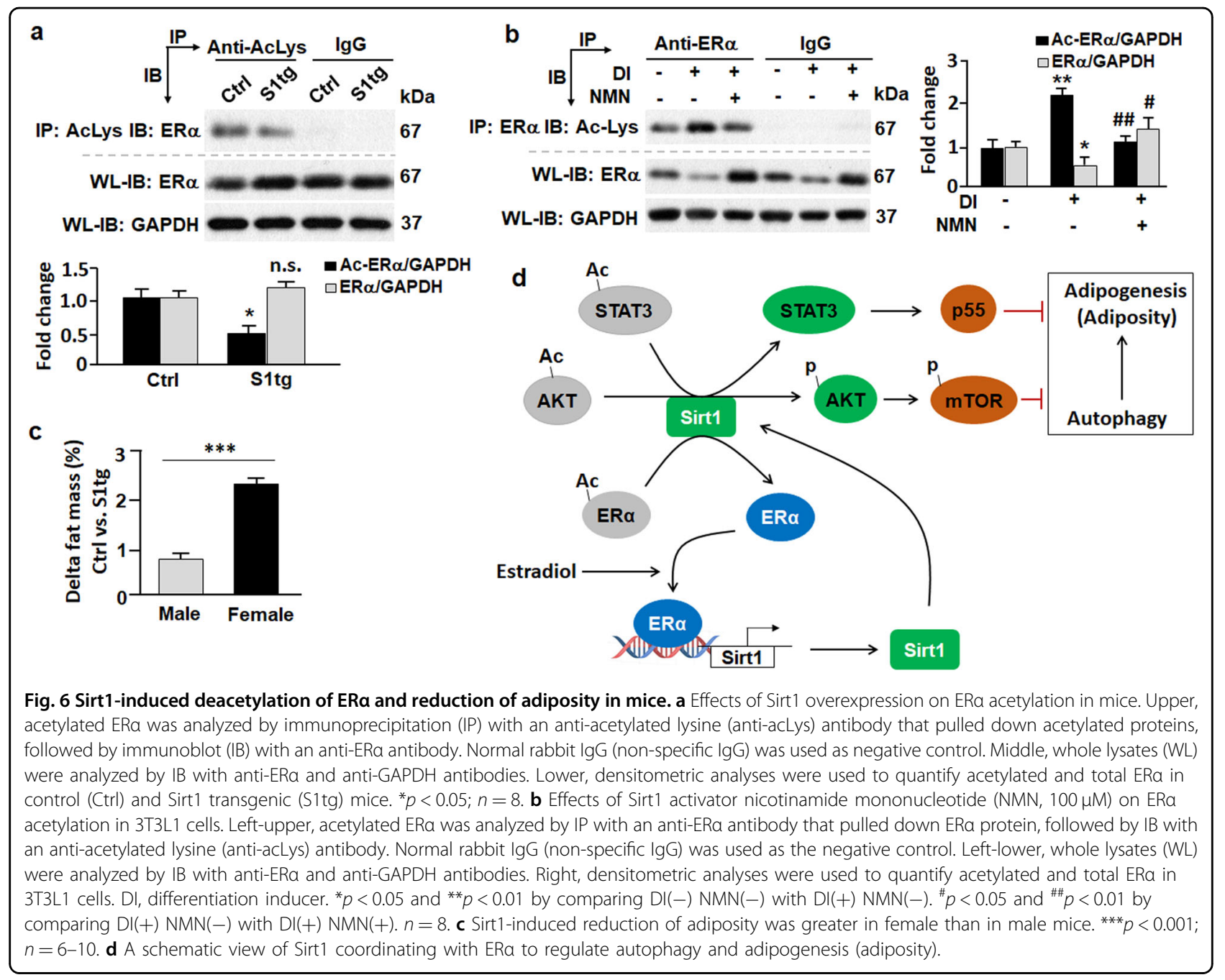

phosphoinositide 3-kinase acts as a direct inhibitor of autophagy by blocking ability of p 85 in autophagy initia$\operatorname{tion}^{42}$. We found that Sirt1 deacetylated and activated STAT3, which drastically induced p55 expression (Fig. 4h, j). Activation of STAT3 and resultant induction of p55 accounts at least in part for the mitigated autophagy in cells or animals overexpressing Sirt1. Of note, Sirt1 appears to constitute a positive feedback with ER $\alpha$ signaling via deacetylation and possibly transcriptional regulation $^{58}$, which warrants further investigation in the future (Fig. 6d). Altogether, our data suggest that Sirt1 serves as a suppressor of autophagy by activating AKT-mTOR and STAT3-p55 cascades, which mediates sex difference in adiposity via crosstalk with E2/ER $\alpha$ signaling.

Our study is the first to document the role of Sirt1 in regulating autophagy in adipose tissue. Sirt1 appeared to act on the initiation of autophagy via inhibitory phosphorylation of ULK1 by mTOR. Notably, Sirt1 overexpression resulted in marginal changes in phosphorylation of mTOR and
ULK1 in skeletal muscle (data not shown), suggesting that Sirt1 plays tissue- or cell type-dependent roles in autophagy. Indeed, Sirt1 induces autophagy by deacetylating and activating Atg5, Atg7, or LC3, but this function was absent in certain cell types ${ }^{28-30,39}$. For instance, deacetylation of LC3 and Atg7 leads to activation of autophagy in spermatids; nevertheless, knockout of Sirt1 attenuated autophagy and spermiogenesis in germ cells but not in steroidogenic cells ${ }^{39}$. It is, therefore, of interest for future studies to explore the molecular and cellular factors that determine the cell typedependent roles for Sirt1 in the regulation of autophagy.

\section{Materials and methods Mice}

The S1tg and control mice were bred and housed as described previously ${ }^{7,20}$. The ER $\alpha \mathrm{KO}\left(\mathrm{ER}^{-1-}\right)$ and control mice $\left(\mathrm{ER \alpha}^{+/+}\right)$were obtained by breeding heterozygous males to females ${ }^{7}$. All the mice were housed in plastic cages on a 12-h light-dark photocycle and with free access to water and regular chow diet ${ }^{7,59}$. At the age 
of 6-8 weeks, the S1tg and control mice were weighed, and fat mass was measured with a Bruker Minispec LF90 NMR Analyzer (Bruker Optics, Billerica, MA, USA), and then euthanized for tissue collection. ER $\alpha \mathrm{KO}$ and control mice were sacrificed for tissue collection at the age of 12-16 weeks. The inguinal WAT pads were collected and weighed quickly and snap freezing in liquid nitrogen. Power calculation suggested that 6 mice per group were required to obtain statistically meaningful data. Mice were randomly grouped for each genotype. Animal use procedures followed the National Institutes of Health guidelines and were approved by the Institutional Animal Care and Use Committees at University of Florida and Virginia Tech.

\section{T3L1 cell culture, differentiation, and treatment}

3T3L1 preadipocytes (ATCC CL-173, Manassas, VA, USA) were cultured in basal media (DMEM media containing $10 \% \mathrm{FBS}, 100$ units $/ \mathrm{ml}$ penicillin and $100 \mu \mathrm{g} / \mathrm{ml}$ streptomycin $(1 \times \mathrm{P} / \mathrm{S}))$, at $37^{\circ} \mathrm{C}$ in a humidified atmosphere of $5 \% \mathrm{CO}_{2}{ }^{31,60}$. The media were replaced every 2 days until the cells became confluent (day 0 ), and after 2 more days (day 2) the media were changed to differentiation medium I (DMEM with $10 \% \mathrm{FBS}, \mathrm{P} / \mathrm{S}(1 \times)$, IBMX $(0.5 \mathrm{mM})$, dexamethasone $(1 \mu \mathrm{M})$, insulin $(1 \mu \mathrm{g} /$ $\mathrm{ml})$, and rosiglitazone $(2 \mu \mathrm{M}))$. At the end of day 4 , the media were changed to differentiation medium II (DMEM with $10 \% \mathrm{FBS}, \mathrm{P} / \mathrm{S}(1 \times)$, and insulin $(1 \mu \mathrm{g} / \mathrm{ml}))$. At the end of day 6 , the media were changed to basal media and the cells were maintained until day 12 . Preadipocytes without differentiation induction were maintained in basal media and supplied with fresh medium every 2 days till day 12 . Resveratrol (RSV) at the concentrations of $0-50 \mu \mathrm{M}$, $\mathrm{NMN}$ at concentration of $100 \mu \mathrm{M}$, and $\beta$-estradiol (E2) of $0.1 \mu \mathrm{M}$, were used to treat cells as indicated. Other chemicals were used at the concentrations established previously, including bafilomycin A1 (4 nM), and leupeptin $(0.4 \mathrm{ng} / \mathrm{ml})^{6}$, to treat the cells during differentiation.

\section{Cell transfection}

After cells reached $80-90 \%$ confluent, overexpression (Sirt1-KI) or knockdown (Sirt1-KD) of Sirt1 was achieved by incubating the cells with adenoviruses carrying GFP (control) and Sirt1 coding sequences, or adenoviruses carrying GFP (GCATCAAGGTGAACTTCAAGA, control) and Sirt1 (GCACCGATCCTCGAACAATTC) shRNA sequences, at 100 multiplicity of infection ${ }^{61}$. After 2 days, cell differentiation was induced as described above and cells were analyzed or harvested on day 9 or day 12 as indicated.

\section{Oil red $O$ staining}

The Oil Red $\mathrm{O}$ working solution was freshly prepared by mixing $0.35 \%$ stock solution with $\mathrm{dH}_{2} \mathrm{O}(6: 4)$ and filtered, and the staining was conducted as described ${ }^{6,33,60}$. After the media were removed, the cells were washed once with cold phosphate buffered saline and fixed in $4 \%$ formaldehyde at room temperature for $10 \mathrm{~min}$. The cells were then washed with $\mathrm{dH}_{2} \mathrm{O}$ and air dried. Oil Red $\mathrm{O}$ working solution was added to start the staining at room temperature for $30 \mathrm{~min}$. The stained cells were washed with $\mathrm{dH}_{2} \mathrm{O}$ for 4 times before the images were captured with a Nikon ECLIPSE Ti Inverted Microscope (Melville, NY, USA).

\section{Autophagy flux assay}

Preadipocytes and mature adipocytes were treated with bafilomycin A1 (inhibitor of autophagosome acidification, at $0.1 \mu \mathrm{M}$ ) plus leupeptin (the inhibitor of lysosomal proteases, at $10 \mu \mathrm{g} / \mathrm{ml}$ ) for $4 \mathrm{~h}$. The cells were then harvested to prepare cell lysates as previously described ${ }^{6,33,60}$. The turnover of LC3-II or p62 protein, i.e., the autophagic removal of the substrate, was measured by Western blotting and image analysis to assess autophagy flux ${ }^{6,31,62}$.

\section{Immunoprecipitation}

Immunoprecipitation was conducted to purify and enrich acetylated ER $\alpha$ to ensure the specificity and sensitivity of detection as described previously: ${ }^{15,63}$ antiacetyl Lysine (ab190479) antibody and protein A/G Sepharose (ab193262) from abcam (Cambridge, MA, USA); ER $\alpha$ (04-820) antibody from EMD Millipore (Billerica, MA, USA); normal rabbit IgG (\#2729) from Cell Signaling Technology (Beverly, MA, USA).

\section{Western blotting}

Tissue and cell lysates were prepared with PLC lysis buffer (30 mM Hepes, pH 7.5, $150 \mathrm{mM} \mathrm{NaCl}, 10 \%$ glycerol, $1 \%$ Triton X-100, $1.5 \mathrm{mM} \mathrm{MgCl}_{2}, 1 \mathrm{mM}$ EGTA, $10 \mathrm{mM} \mathrm{NaPPi}, 100 \mathrm{mM} \mathrm{NaF}, 1 \mathrm{mM} \mathrm{Na} \mathrm{VO}_{4}$ ) supplemented with protease inhibitor cocktail (Roche), and $1 \mathrm{mM} \mathrm{PMSF}{ }^{6,31,60}$. Total protein concentrations of the lysates were determined using a DC protein assay kits (Bio-Rad). Antibody (catalog number) information: GAPDH (MA5-15738) and $\beta$-actin (MA5-15739) antibodies were purchased from Pierce (Rockford, IL, USA); p-mTOR (Ser2448) antibody (5536s) and p-ULK1(Ser757) antibody (14202s) from Cell Signaling Technology (Beverly, MA, USA); ER $\alpha$ (04-820) and Sirt1 (07-131) antibodies from EMD Millipore (Billerica, MA, USA); p62 (ab56416) antibody from abcam (Cambridge, MA, USA); and LC3B antibody (L7543) from Sigma (Billerica, MA, USA).

\section{Statistical analysis}

Measurements were duplicated or triplicated, with 6-10 mice included in each group. Data were presented as mean \pm SD. Unless the use of female mice were specified, 
the animal studies were conducted on males. Differences between groups and treatments were validated by oneway analysis of variance or a two-sided $t$-test. A value of $p<0.05$ was considered statistically significant.

\section{Acknowledgements}

This work was supported in part by the American Heart Association Grant (18TPA34230082 to Z.C.), and the USDA National Institute of Food and Agriculture Grant (1020373 to Z.C).

\section{Author details}

'Department of Human Nutrition, Foods, and Exercise, Virginia Tech, Blacksburg, VA 24061, USA. 'Food Science and Human Nutrition Department, University of Florida, Gainesville, FL 32611, USA. ${ }^{3}$ Institute for Cancer Genetics, and Department of Pathology and Cell Biology, and Herbert Irving Comprehensive Cancer Center, College of Physicians and Surgeons, Columbia University, New York, NY 10032, USA. ${ }^{4}$ Department of Biochemistry and Molecular Biology, Indiana University School of Medicine, Indianapolis, IN 46202, USA. ${ }^{5}$ Department of Surgery, Division of Urology, Beth Israel Deaconess Medical Center, Harvard Medical School, Boston, MA 02115, USA. ${ }^{6}$ Present address: Cutaneous Biology Research Center, Massachusetts General Hospital, Harvard Medical School, Charlestown, MA 02129, USA

\section{Author contributions}

Z.C. conceived the study, Z.C. and Z.T. wrote the paper, Z.T., L.S., J.P., and L.Z. performed experiments and analyzed data with Z.C., W.G., and X.C.D. provided viral vectors and S1tg mice, respectively. Z.T., L.S., J.P., L.Z., W.G., X.D., D.L., Z.W., A.O., and Z.C. communicated for data interpretation and paper writing and revision.

\section{Conflict of interest}

The authors declare no competing interests.

\section{Publisher's note}

Springer Nature remains neutral with regard to jurisdictional claims in published maps and institutional affiliations.

Supplementary information The online version contains supplementary material available at https://doi.org/10.1038/s41420-021-00438-8.

Received: 1 December 2020 Revised: 25 January 2021 Accepted: 14 February 2021

Published online: 15 March 2021

\section{References}

1. Mizushima, N. \& Komatsu, M. Autophagy: renovation of cells and tissues. Cell 147, 728-741 (2011).

2. Boya, P., Codogno, P. \& Rodriguez-Muela, N. Autophagy in stem cells: repair, remodelling and metabolic reprogramming. Development 145, https:/doi. org/10.1242/dev.146506 (2018).

3. Cheng, Z. The FoxO-autophagy axis in health and disease. Trends Endocrinol. Metab.: TEM 30, 658-671 (2019).

4. Singh, R. et al. Autophagy regulates adipose mass and differentiation in mice. J. Clin. Investig. 119, 3329-3339 (2009).

5. Zhang, Y. et al. Adipose-specific deletion of autophagy-related gene 7 (atg7) in mice reveals a role in adipogenesis. Proc. Natl Acad. Sci. USA 106, 19860-19865 (2009).

6. Liu, L. et al. FoxO1 antagonist suppresses autophagy and lipid droplet growth in adipocytes. Cell Cycle 15, 2033-2041 (2016).

7. Tao, Z. et al. Estradiol signaling mediates gender difference in visceral adiposity via autophagy. Cell Death Dis. 9, 309 (2018).

8. Romero, M. et al. TP53INP2 regulates adiposity by activating beta-catenin through autophagy-dependent sequestration of GSK3beta. Nat. Cell Biol. 20, 443-454 (2018).
9. Haim, Y. et al. Elevated autophagy gene expression in adipose tissue of obese humans: a potential non-cell-cycle-dependent function of E2F1. Autophagy 11, 2074-2088 (2015).

10. Kosacka, J. et al. Autophagy in adipose tissue of patients with obesity and type 2 diabetes. Mol. Cell. Endocrinol. 409, 21-32 (2015).

11. Soussi, H. et al. DAPK2 downregulation associates with attenuated adipocyte autophagic clearance in human obesity. Diabetes 64, 3452-3463 (2015).

12. Mizunoe, $Y$. et al. Involvement of lysosomal dysfunction in autophagosome accumulation and early pathologies in adipose tissue of obese mice. Autophagy 13, 642-653 (2017).

13. Cai, J. et al. Autophagy ablation in adipocytes induces insulin resistance and reveals roles for lipid peroxide and Nrf2 signaling in adipose-liver crosstalk. Cell Rep. 25, 1708-1717.e1705 (2018).

14. Chang, H. C. \& Guarente, L. SIRT1 and other sirtuins in metabolism. Trends Endocrinol. Metab.: TEM 25, 138-145 (2014).

15. Cheng, Z. et al. Foxo1 integrates insulin signaling with mitochondrial function in the liver. Nat. Med. 15, 1307-1311 (2009).

16. Lagouge, M. et al. Resveratrol improves mitochondrial function and protects against metabolic disease by activating SIRT1 and PGC-1alpha. Cell 127, 1109-1122 (2006).

17. Feige, J. N. et al. Specific SIRT1 activation mimics low energy levels and protects against diet-induced metabolic disorders by enhancing fat oxidation. Cell Metab. 8, 347-358 (2008).

18. Milne, J. C. et al. Small molecule activators of SIRT1 as therapeutics for the treatment of type 2 diabetes. Nature 450, 712-716 (2007).

19. Pfluger, P. T., Herranz, D., Velasco-Miguel, S., Serrano, M. \& Tschop, M. H. Sirt1 protects against high-fat diet-induced metabolic damage. Proc. Natl Acad. Sci. USA 105, 9793-9798 (2008).

20. Banks, A. S. et al. SirT1 gain of function increases energy efficiency and prevents diabetes in mice. Cell Metab. 8, 333-341 (2008).

21. Boutant, M. et al. SIRT1 gain of function does not mimic or enhance the adaptations to intermittent fasting. Cell Rep. 14, 2068-2075 (2016).

22. Boutant, M. et al. SIRT1 enhances glucose tolerance by potentiating brown adipose tissue function. Mol. Metab. 4, 118-131 (2015).

23. Chattopadhyay, T. et al. Spatiotemporal gating of SIRT1 functions by OGlcNAcylation is essential for liver metabolic switching and prevents hyperglycemia. Proc. Natl Acad. Sci. USA 117, 6890-6900 (2020).

24. Picard, F. et al. Sirt1 promotes fat mobilization in white adipocytes by repressing PPAR-gamma. Nature 429, 771-776 (2004).

25. Mayoral, R. et al. Adipocyte SIRT1 knockout promotes PPARgamma activity, adipogenesis and insulin sensitivity in chronic-HFD and obesity. Mol. Metab. 4 378-391 (2015).

26. Chalkiadaki, A. \& Guarente, L. High-fat diet triggers inflammation-induced cleavage of SIRT1 in adipose tissue to promote metabolic dysfunction. Cell Metab. 16, 180-188 (2012).

27. Qiang, L. et al. Brown remodeling of white adipose tissue by SirT1-dependent deacetylation of Ppargamma. Cell 150, 620-632 (2012).

28. Ou, X., Lee, M. R., Huang, X., Messina-Graham, S. \& Broxmeyer, H. E. SIRT1 positively regulates autophagy and mitochondria function in embryonic stem cells under oxidative stress. Stem Cells 32, 1183-1194 (2014).

29. Yao, Z. Q. et al. A novel small-molecule activator of Sirtuin-1 induces autophagic cell death/mitophagy as a potential therapeutic strategy in glioblastoma. Cell Death Dis. 9, 767 (2018).

30. Lee, I. H. et al. A role for the NAD-dependent deacetylase Sirt1 in the regulation of autophagy. Proc. Natl Acad. Sci. USA 105, 3374-3379 (2008).

31. Tao, Z., Liu, L., Zheng, L. D. \& Cheng, Z. Autophagy in adipocyte differentiation. Methods Mol. Biol. 1854, 45-53 (2019).

32. Bjørkøy, G. et al. p62/SQSTM1 forms protein aggregates degraded by autophagy and has a protective effect on huntingtin-induced cell death. J. Cell Biol. 171, 603-614 (2005).

33. Liu, L. et al. FoxO1 interacts with transcription factor EB and differentially regulates mitochondrial uncoupling proteins via autophagy in adipocytes. Cell Death Discov. 2, 16066 (2016).

34. Hansen, M., Rubinsztein, D. C. \& Walker, D. W. Autophagy as a promoter of longevity: insights from model organisms. Nat. Rev. Mol. Cell Biol. 19, 579-593 (2018).

35. Rosner, M., Siegel, N., Valli, A., Fuchs, C. \& Hengstschlager, M. mTOR phosphorylated at $\mathbf{S 2 4 4 8}$ binds to raptor and rictor. Amino Acids 38, 223-228 (2010). 
36. Reynolds, T. H. T., Bodine, S. C. \& Lawrence, J. C. Jr. Control of Ser2448 phosphorylation in the mammalian target of rapamycin by insulin and skeletal muscle load. J. Biol. Chem. 277, 17657-17662 (2002).

37. Kim, Y. C. \& Guan, K. L. mTOR: a pharmacologic target for autophagy regulation. J. Clin. Investig. 125, 25-32 (2015).

38. Foster, K. G. \& Fingar, D. C. Mammalian target of rapamycin (mTOR): conducting the cellular signaling symphony. J. Biol. Chem. 285, 14071-14077 (2010).

39. Liu, $\mathrm{C}$. et al. Sirt1 regulates acrosome biogenesis by modulating autophagic flux during spermiogenesis in mice. Development 144, 441-451 (2017).

40. Sundaresan, N. R. et al. The deacetylase SIRT1 promotes membrane localization and activation of Akt and PDK1 during tumorigenesis and cardiac hypertrophy. Sci. Signal. 4, ra46 (2011).

41. Pensa, S. et al. The PI3K regulatory subunits $\mathrm{p} 55 \mathrm{al}$ pha and $\mathrm{p} 50 \mathrm{al}$ pha regulate cell death in vivo. Cell Death Differ. 21, 1442-1450 (2014)

42. Pensa, S. et al. Signal transducer and activator of transcription 3 and the phosphatidylinositol 3-kinase regulatory subunits p55alpha and p50alpha regulate autophagy in vivo. FEBS J. 281, 4557-4567 (2014).

43. Schenk, S. et al. Sirt1 enhances skeletal muscle insulin sensitivity in mice during caloric restriction. J. Clin. Investig. 121, 4281-4288 (2011).

44. Nave, B. T., Ouwens, M., Withers, D. J., Alessi, D. R. \& Shepherd, P. R. Mammalian target of rapamycin is a direct target for protein kinase B: identification of a convergence point for opposing effects of insulin and amino-acid deficiency on protein translation. Biochemical J. 344(Pt 2), 427-431 (1999).

45. Sekulic, A. et al. A direct linkage between the phosphoinositide 3-kinase-AKT signaling pathway and the mammalian target of rapamycin in mitogenstimulated and transformed cells. Cancer Res. 60, 3504-3513 (2000).

46. Chiang, G. G. \& Abraham, R. T. Phosphorylation of mammalian target of rapamycin (mTOR) at Ser-2448 is mediated by p70S6 kinase. J. Biol. Chem. 280, 25485-25490 (2005).

47. Holz, M. K. \& Blenis, J. Identification of S6 kinase 1 as a novel mammalian target of rapamycin (mTOR)-phosphorylating kinase. J. Biol. Chem. 280, 26089-26093 (2005).

48. Heine, P. A., Taylor, J. A., Iwamoto, G. A., Lubahn, D. B. \& Cooke, P. S. Increased adipose tissue in male and female estrogen receptor-alpha knockout mice. Proc. Natl Acad. Sci. USA 97, 12729-12734 (2000).
49. Davis, K. E. et al. The sexually dimorphic role of adipose and adipocyte estrogen receptors in modulating adipose tissue expansion, inflammation, and fibrosis. Mol. Metab. 2, 227-242 (2013).

50. Das, A. et al. Impairment of an endothelial $\mathrm{NAD}(+)-\mathrm{H} 2 \mathrm{~S}$ signaling network is a reversible cause of vascular aging. Cell 173, 74-89 e20 (2018).

51. Song, J. et al. Nicotinamide mononucleotide promotes osteogenesis and reduces adipogenesis by regulating mesenchymal stromal cells via the SIRT1 pathway in aged bone marrow. Cell Death Dis. 10, 336 (2019).

52. Hutson, D. D. et al. Estrogen receptor profiles across tissues from male and female Rattus norvegicus. Biol. Sex. Differ. 10, 4 (2019).

53. Saito, T. et al. Estrogen contributes to gender differences in mouse ventricular repolarization. Circulation Res. 105, 343-352 (2009).

54. Lumish, H. S., O'Reilly, M. \& Reilly, M. P. Sex differences in genomic drivers of adipose distribution and related cardiometabolic disorders: opportunities for precision medicine. Arterioscler. Thromb. Vasc. Biol. 40, 45-60 (2020).

55. Chang, E., Varghese, M. \& Singer, K. Gender and sex differences in adipose tissue. Curr. Diab. Rep. 18, 69 (2018).

56. Elangovan, S. et al. SIRT1 is essential for oncogenic signaling by estrogen/ estrogen receptor alpha in breast cancer. Cancer Res. 71, 6654-6664 (2011).

57. Kreuzaler, P. A. et al. Stat3 controls lysosomal-mediated cell death in vivo. Nat. Cell Biol. 13, 303-309 (2011).

58. Yao, Y., Li, H., Gu, Y., Davidson, N. E. \& Zhou, Q. Inhibition of SIRT1 deacetylase suppresses estrogen receptor signaling. Carcinogenesis 31, 382-387 (2010).

59. Liu, L. et al. Tamoxifen reduces fat mass by boosting reactive oxygen species. Cell Death Dis. 6, e1586 (2015).

60. Zou, P. et al. Targeting FoxO1 with AS1842856 suppresses adipogenesis. Cell Cycle 13, 3759-3767 (2014).

61. Wei, D., Tao, R., Zhang, Y., White, M. F. \& Dong, X. C. Feedback regulation of hepatic gluconeogenesis through modulation of SHP/NrOb2 gene expression by Sirt1 and FoxO1. Am. J. Physiol. Endocrinol. Metab. 300, E312-E320 (2011).

62. Mizushima, N., Yoshimori, T. \& Levine, B. Methods in mammalian autophagy research. Cell 140, 313-326 (2010).

63. Sadagurski, M. et al. IRS2 increases mitochondrial dysfunction and oxidative stress in a mouse model of Huntington disease. J. Clin. Investig. 121, 4070-4081 (2011) 